\title{
Pembrolizumab alone or in combination with chemotherapy as first- line therapy for patients with advanced gastric or gastroesophageal junction adenocarcinoma: results from the phase II nonrandomized KEYNOTE-059 study
}

\author{
Yung-Jue Bang ${ }^{1}$ Yoon-Koo Kang ${ }^{2}$. Daniel V. Catenacci ${ }^{3} \cdot K^{2}$ Muro ${ }^{4} \cdot$ Charles S. Fuchs $^{5} \cdot$ Ravit Geva $^{6} \cdot$ Hiroki Hara $^{7}$. \\ Talia Golan $^{8}$. Marcelo Garrido ${ }^{9}$. Shadia I. Jalal ${ }^{10}$. Christophe Borg ${ }^{11} \cdot$ Toshihiko Doi $^{12} \cdot$ Harry H. Yoon $^{13}$. \\ Mary J. Savage $^{14}$. Jiangdian Wang ${ }^{14} \cdot$ Rita P. Dalal $^{14}$. Sukrut Shah ${ }^{14} \cdot$ Zev A. Wainberg ${ }^{15} \cdot$ Hyun Cheol Chung $^{16}$
}

Received: 5 September 2018 / Accepted: 1 December 2018 / Published online: 25 March 2019

(c) The Author(s) 2019

\begin{abstract}
Background The multicohort, phase II, nonrandomized KEYNOTE-059 study evaluated pembrolizumab \pm chemotherapy in advanced gastric/gastroesophageal junction cancer. Results from cohorts 2 and 3, evaluating first-line therapy, are presented. Methods Patients $\geq 18$ years old had previously untreated recurrent or metastatic gastric/gastroesophageal junction adenocarcinoma. Cohort 3 (monotherapy) had programmed death receptor 1 combined positive score $\geq 1$. Cohort 2 (combination therapy) received pembrolizumab $200 \mathrm{mg}$ on day 1 , cisplatin $80 \mathrm{mg} / \mathrm{m}^{2}$ on day 1 (up to 6 cycles), and 5-fluorouracil $800 \mathrm{mg} / \mathrm{m}^{2}$ on days $1-5$ of each 3 -week cycle (or capecitabine $1000 \mathrm{mg} / \mathrm{m}^{2}$ twice daily in Japan). Primary end points were safety (combination therapy) and objective response rate per Response Evaluation Criteria in Solid Tumors version 1.1 by central review, and safety (monotherapy).

Results In the combination therapy and monotherapy cohorts, 25 and 31 patients were enrolled; median follow-up was 13.8 months (range 1.8-24.1) and 17.5 months (range 1.7-20.7), respectively. In the combination therapy cohort, grade 3/4 treatment-related adverse events occurred in 19 patients (76.0\%); none were fatal. In the monotherapy cohort, grade 3-5 treatment-related adverse events occurred in seven patients (22.6\%); one death was attributed to a treatment-related adverse event (pneumonitis). The objective response rate was 60.0\% [95\% confidence interval (CI), 38.7-78.9] (combination therapy) and $25.8 \%$ (95\% CI 11.9-44.6) (monotherapy).

Conclusions Pembrolizumab demonstrated antitumor activity and was well tolerated as monotherapy and in combination with chemotherapy in patients with previously untreated advanced gastric/gastroesophageal junction adenocarcinoma.

Clinical Trial ClinicalTrials.gov NCT02335411
\end{abstract}

Keywords Pembrolizumab · Cisplatin · 5-Fluorouracil · Capecitabine · Gastric cancer

Rita P. Dalal was affiliated with Medical Oncology, Merck \& Co., Inc., Kenilworth, NJ, USA, at the time of the study.

Yung-Jue Bang, Yoon-Koo Kang and Hyun Cheol Chung contributed equally.

Electronic supplementary material The online version of this article (https://doi.org/10.1007/s10120-018-00909-5) contains supplementary material, which is available to authorized users.

Yung-Jue Bang

bangyj@snu.ac.kr

Extended author information available on the last page of the article

\section{Introduction}

Gastric cancer is the fifth most common malignancy worldwide. In 2012, it led to 723,000 deaths [1]. Most cases are advanced at diagnosis, and prognosis is poor [2, 3]. Chemotherapy containing fluoropyrimidine plus a platinum agent is standard first-line treatment for advanced gastric or gastroesophageal junction (G/GEJ) adenocarcinoma not expressing human epidermal growth factor receptor 2 (HER2) [4-6]. In previously untreated gastric cancer, this regimen demonstrated objective response rates (ORRs) of $25-48 \%$ and median overall survival (OS) of 8-11.2 months [7-12]. 
Tumor cells frequently use the programmed death receptor 1 (PD-1) pathway to evade immune surveillance [13, 14]. Binding of PD-1 to its ligands, PD-L1 and PD-L2, inhibits effector T-cell function, resulting in suppression of antitumor immune response [13, 14]. PD-L1 is frequently upregulated in gastric cancer [15-19]. Pembrolizumab is a selective, humanized, immunoglobulin G4א monoclonal antibody that blocks interactions between PD-1 and its ligands [20]. The phase II KEYNOTE-059 clinical trial, a multicohort study designed to evaluate pembrolizumab alone or in combination with chemotherapy in patients with recurrent or metastatic G/GEJ adenocarcinoma, included a cohort of patients whose disease had progressed while they previously received at least two systemic therapies for advanced disease (cohort 1; pembrolizumab monotherapy) and two cohorts of patients who had not previously received systemic therapy for advanced disease. Data from cohort 1 have already been published and demonstrate durable responses with pembrolizumab monotherapy, with higher ORR and longer response durations in patients with PD-L1-positive tumors [combined positive score (CPS) $\geq 1$ ] than with PD-L1-negative tumors [21]. Based on these results, the US Food and Drug Administration approved pembrolizumab for the treatment of patients with recurrent or metastatic G/GEJ adenocarcinoma whose tumors express PD-L1 and whose disease has progressed on or after $\geq 2$ prior lines of therapy [20]. In the phase III, KEYNOTE-061 study, pembrolizumab did not significantly improve OS, versus paclitaxel, as second-line therapy in patients with advanced G/GEJ cancer and PD-L1 CPS $\geq 1$ [22].

Chemotherapeutic agents such as cisplatin and 5-fluorouracil enhance immunogenicity of cancer cells and increase susceptibility to immune-mediated cytotoxicity [23-25]. Combining pembrolizumab with chemotherapy has demonstrated efficacy and manageable safety in other cancers $[26,27]$. Cohorts 2 and 3 of the KEYNOTE-059 study were designed to further explore pembrolizumab in combination therapy and as monotherapy in the PD-L1-positive population. Herein, we present results from cohorts 2 and 3 of the KEYNOTE-059 study, which evaluated first-line therapy with pembrolizumab plus chemotherapy (cohort 2) or alone (cohort 3 ) in advanced G/GEJ cancer.

\section{Materials and methods}

KEYNOTE-059 is a multicenter, open-label, nonrandomized, 3-cohort, phase II study. Results for cohorts 2 and 3 are presented; results for cohort 1 were published separately [21]. The study was conducted in accordance with Good Clinical Practice guidelines and the Declaration of Helsinki. The protocol was approved by institutional review boards and independent ethics committees for each institution. All patients provided written, informed consent. This trial was registered with ClinicalTrials.gov, number NCT02335411.

\section{Patients}

Eligibility criteria for both cohorts were age $\geq 18$ years, histologically or cytologically confirmed recurrent or metastatic G/GEJ adenocarcinoma, no prior systemic therapy for advanced disease, measurable disease per Response Evaluation Criteria in Solid Tumors version 1.1 (RECIST v1.1) by central review, HER2/neu-negative status, Eastern Cooperative Oncology Group performance status 0/1, life expectancy $\geq 3$ months, and adequate organ function.

For cohort 2, patients had to provide new or archival tumor tissue for PD-L1 analysis. For cohort 3, patients had to have PD-L1-positive tumors as per the PD-L1 IHC 22C3 pharmDx assay (Agilent Technologies, Carpinteria, CA, USA). PD-L1 positivity was defined as CPS $\geq 1$, where CPS is the number of PD-L1-positive cells (tumor cells, lymphocytes, and macrophages) divided by the total number of tumor cells multiplied by 100 [21]. Exclusion criteria are presented in Online Resource Table S1. We assessed DNA mismatch repair across 5 mononucleotide repeat markers (NR21, NR24, BAT25, BAT26, MONO27) using DNA extracted from formalin-fixed, paraffin-embedded tumor samples and blood (normal control) using the MSI Analysis System, version 1.2 (Promega). In microsatellite instabilityhigh tumors, 2 or more markers were changed, compared with normal controls.

\section{Study treatment}

In cohort 2, patients received pembrolizumab $200 \mathrm{mg}$ by intravenous infusion over $30 \mathrm{~min}$ on day 1, cisplatin $80 \mathrm{mg} /$ $\mathrm{m}^{2}$ on day 1 (for up to 6 cycles), and 5-fluorouracil $800 \mathrm{mg} /$ $\mathrm{m}^{2}$ by continuous infusion on days $1-5$ of each 21 -day cycle. In Japan only, patients received capecitabine $1000 \mathrm{mg} / \mathrm{m}^{2}$ twice daily orally on days 1-14 of each 21-day cycle instead of 5-fluorouracil. Up to two dose reductions were allowed per chemotherapy agent (Online Resource Table S2). In cohort 3 , patients received pembrolizumab monotherapy $200 \mathrm{mg}$ by intravenous infusion over $30 \mathrm{~min}$ on day 1 of each 21-day cycle.

Pembrolizumab dose reduction was not permitted. Treatment was continued for up to 35 cycles ( 2 years) or until confirmed disease progression, unacceptable toxicity, complete response, or patient or investigator decision to withdraw. Clinically stable patients with radiographic progressive disease (PD) could continue treatment at the investigator's discretion until confirmed PD. Patients in cohort 2 who discontinued chemotherapy could continue pembrolizumab if they had not met criteria for discontinuation. 


\section{Outcomes and assessments}

For cohort 2, the primary end points were safety and tolerability. Secondary end points included ORR (percentage of patients who experienced complete or partial response) per RECIST v1.1 by central imaging review and evaluation of the relationship between PD-L1 expression and efficacy. For cohort 3, primary end points were ORR and safety and tolerability. Secondary end points included disease control rate (DCR; percentage of patients who experienced complete or partial response or stable disease for $\geq 6$ months), duration of response, progression-free survival (PFS; time from study treatment initiation to first documented instance of PD or death) per RECIST v1.1 by central imaging review, and OS (time from initiation of study treatment to death from any cause).

Tumors were assessed by computed tomography or magnetic resonance imaging at 9 weeks after study treatment initiation, every 6 weeks for year 1 , and every 9 weeks thereafter (or more often if clinically indicated). Response was confirmed by repeat radiographic assessment $\geq 4$ weeks after first documentation. Patients were followed up every 12 weeks for survival status until death, withdrawal of consent, or study end.

Patients were monitored for adverse events (AEs) until 30 days after the last dose of treatment (90 days for serious AEs and events of clinical interest). AEs were graded using National Cancer Institute Common Terminology Criteria for Adverse Events, version 4.0; investigators determined treatment attribution.

\section{Statistical analysis}

For cohort 2, the sample size was based on clinical experience. A sample size of $\sim 18$ patients was planned to provide the reasonable two-sided $95 \%$ confidence interval (CI) of AE incidence (Online Resource Table S3). For cohort 3 , a sample size of $\sim 25$ patients with PD-L1 CPS $\geq 1$ was estimated using Bayesian sample size methods assuming a uniform prior for the response rate and a true ORR of $<40 \%$ (ORR based on standard of care). Safety and efficacy were analyzed in all patients who received $\geq 1$ dose of study treatment. Safety was analyzed using descriptive statistics. Analyses were conducted using SAS statistical software version 9.3 (SAS Institute, Inc). ORR and DCR with $95 \%$ CI were calculated using the exact method based on binomial distribution (Clopper-Pearson method [28]). Patients with missing data were considered nonresponders. Time-to-event end points were estimated using the Kaplan-Meier method, with censoring at the last date of assessment for patients with missing data.

\section{Results}

The study was conducted at 57 sites in 17 countries. For cohort 2, 25 patients were enrolled at 10 sites in the Republic of Korea (South Korea), Japan, Israel, the United States, and France between March 2, 2015, and December 14, 2015. For cohort 3, 31 patients were enrolled at 18 sites in Canada, Chile, Israel, Japan, South Korea, and the United States between July 23, 2015, and December 2, 2015. Patient characteristics are presented in Table 1.

At data cutoff (April 21, 2017), median follow-up was 13.8 months (range 1.8-24.1) in cohort 2 and 17.5 months (range 1.7-20.7) in cohort 3. In cohort 2, 22 patients $(88.0 \%)$ had discontinued study treatment and three $(12.0 \%)$ remained on treatment. In cohort 3, 24 patients (77.4\%) had discontinued study treatment, whereas seven patients (22.6\%) remained on treatment. In both cohorts,

Table 1 Patient characteristics

\begin{tabular}{|c|c|c|}
\hline Characteristic & $\begin{array}{l}\text { Cohort } 2 \\
N=25\end{array}$ & $\begin{array}{l}\text { Cohort } 3 \\
N=31\end{array}$ \\
\hline Male & $16(64.0)$ & $19(61.3)$ \\
\hline Age, median (range) (years) & $64(21-82)$ & $62(32-75)$ \\
\hline \multicolumn{3}{|l|}{ Race } \\
\hline Asian & $17(68.0)$ & $15(48.4)$ \\
\hline White & $8(32.0)$ & $16(51.6)$ \\
\hline \multicolumn{3}{|l|}{ Region } \\
\hline United States & $3(12.0)$ & $12(38.7)$ \\
\hline East Asia & $16(64.0)$ & $13(41.9)$ \\
\hline Rest of world & $6(24.0)$ & $6(19.4)$ \\
\hline \multicolumn{3}{|l|}{ ECOG performance status } \\
\hline 0 & $15(60.0)$ & $14(45.2)$ \\
\hline 1 & $10(40.0)$ & $17(54.8)$ \\
\hline \multicolumn{3}{|l|}{ Metastatic stage } \\
\hline M0 & $1(4.0)$ & $5(16.1)$ \\
\hline M1 & $24(96.0)$ & $26(83.9)$ \\
\hline Prior surgery for gastric cancer & $5(20.0)$ & $12(38.7)$ \\
\hline \multicolumn{3}{|l|}{ Histology, WHO classification } \\
\hline Tubular adenocarcinoma & $22(88.0)$ & $25(80.6)$ \\
\hline Signet ring cell carcinoma & $2(8.0)$ & $3(9.7)$ \\
\hline Mixed carcinoma & $1(4.0)$ & $2(6.5)$ \\
\hline Other poorly cohesive carcinoma & 0 & $1(3.2)$ \\
\hline \multicolumn{3}{|l|}{ PD-L1 expression } \\
\hline $\mathrm{CPS} \geq 1$ & $16(64.0)$ & $31(100.0)$ \\
\hline $\mathrm{CPS}<1$ & $8(32.0)$ & 0 \\
\hline Unknown & $1(4.0)$ & 0 \\
\hline
\end{tabular}

Unless otherwise indicated, all data are $n(\%)$

$C P S$ combined positive score, ECOG Eastern Cooperative Oncology Group, $P D-L 1$ programmed death ligand 1, WHO World Health Organization 
the most common reason for treatment discontinuation was PD (Online Resource Figure S1, CONSORT).

\section{Treatment exposure and safety}

\section{Cohort 2}

Median duration of pembrolizumab exposure was 7.1 months (range 0.8-23.8), and median number of administrations was 10 (range 2-35). All patients experienced treatment-related AEs (TRAEs) (Table 2). Grade 3 TRAEs occurred in 15 patients $(60.0 \%)$. One TRAE was grade 4 (decreased neutrophil count, 16.0\%). No TRAEs were fatal. AEs and serious AEs related to chemotherapy and pembrolizumab are presented in Online Resource Tables S4 and S5. TRAEs leading to treatment interruption are described in Online Resource Table S6. Three patients (12.0\%) discontinued treatment because of chemotherapy-related AEs: grade 3 stomatitis, grade 2 hypoacusis, and grade 1 increased creatinine level. No patient discontinued treatment because of pembrolizumab-related AEs. Immune-mediated AEs (regardless of attribution) occurred in 12 patients (48.0\%) and were grade 3 in four patients (16.0\%) (Online Resource Table S7). No grade 4/5 immune-mediated AEs occurred. Five of 12 patients who experienced an immune-mediated $\mathrm{AE}(41.7 \%)$ received systemic corticosteroids.

\section{Cohort 3}

Median duration of exposure to pembrolizumab was 2.8 months (range 0.7-20.3), and median number of administrations was five (range 2-30). Overall, TRAEs occurred in 24 patients (77.4\%) (Table 3). Grade 3-5 TRAEs occurred in seven patients (22.6\%). One patient died because of pneumonitis 74 days after the last pembrolizumab dose. Serious TRAEs occurred in six patients (19.4\%). TRAEs resulted in treatment interruption in eight patients (25.8\%) (Online Resource Table S8). No patient discontinued because of a TRAE. Immune-mediated AEs occurred in 10 patients $(32.3 \%)$ and were grade $3-5$ in three patients $(9.7 \%)$ (Online Resource Table S9). Four of ten patients who experienced an immune-mediated $\mathrm{AE}(40.0 \%)$ received systemic corticosteroids.

\section{Antitumor activity}

\section{Cohort 2}

Confirmed ORR was 60.0\% (95\% CI 39.0-79.0), with complete response in one patient (4.0\%) (Table 4). Median time to response was 2.1 months (range 1.9-3.7) and median response duration was 4.6 months (range 2.6-20.3+). At data cutoff, response was ongoing in two of 15 responders
Table 2 Treatment-related AEs of any grade occurring in $\geq 10 \%$ of patients and grade 3 treatment-related AEs occurring in $\geq 1$ patients in cohort 2

\begin{tabular}{|c|c|c|}
\hline \multirow[t]{2}{*}{ Treatment-related AEs ${ }^{\mathrm{a}} n(\%)$} & \multicolumn{2}{|l|}{$\begin{array}{l}\text { Cohort } 2 \\
N=25\end{array}$} \\
\hline & Any grade & Grade 3 \\
\hline Any & $25(100.0)$ & $15(60.0)$ \\
\hline \multicolumn{3}{|l|}{ Hematologic } \\
\hline Decreased neutrophil count & $21(84.0)$ & $12(48.0)$ \\
\hline Anemia & $5(20.0)$ & $2(8.0)$ \\
\hline Decreased platelet count & $8(32.0)$ & $2(8.0)$ \\
\hline Decreased WBC count & $4(16.0)$ & $1(4.0)$ \\
\hline Febrile neutropenia & $1(4.0)$ & $1(4.0)$ \\
\hline \multicolumn{3}{|l|}{ Nonhematologic } \\
\hline Nausea & $13(52.0)$ & $1(4.0)$ \\
\hline Stomatitis ${ }^{\mathrm{b}}$ & $13(52.0)$ & $5(20.0)$ \\
\hline Decreased appetite & $11(44.0)$ & $2(8.0)$ \\
\hline Fatigue & $8(32.0)$ & $2(8.0)$ \\
\hline Diarrhea & $8(32.0)$ & 0 \\
\hline Dysgeusia & $7(28.0)$ & 0 \\
\hline Constipation & $6(24.0)$ & 0 \\
\hline Vomiting & $6(24.0)$ & 0 \\
\hline Hiccups & $5(20.0)$ & 0 \\
\hline Malaise & $5(20.0)$ & 0 \\
\hline $\begin{array}{l}\text { Palmar-plantar erythrodysesthesia } \\
\text { syndrome }\end{array}$ & $4(16.0)$ & $2(8.0)$ \\
\hline Alopecia & $4(16.0)$ & 0 \\
\hline Peripheral sensory neuropathy & $4(16.0)$ & 0 \\
\hline Hyperthyroidism & $4(16.0)$ & 0 \\
\hline Maculopapular rash or rash & $4(16.0)$ & $2(8.0)$ \\
\hline Pyrexia & $3(12.0)$ & $1(4.0)$ \\
\hline Decreased weight & $3(12.0)$ & $1(4.0)$ \\
\hline Increased blood creatinine level & $3(12.0)$ & 0 \\
\hline Mucosal inflammation ${ }^{c}$ & $3(12.0)$ & 0 \\
\hline Peripheral neuropathy & $3(12.0)$ & 0 \\
\hline Increased weight & $3(12.0)$ & 0 \\
\hline Hypophosphatemia & $1(4.0)$ & $1(4.0)$ \\
\hline Polymyalgia rheumatica & $1(4.0)$ & $1(4.0)$ \\
\hline
\end{tabular}

$A E$ adverse event, $W B C$ white blood cell

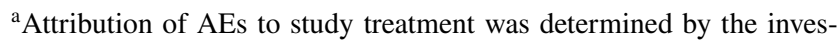
tigator

${ }^{\mathrm{b}}$ Stomatitis refers to inflammation of the mouth and lips

${ }^{\mathrm{c}}$ Mucosal inflammation refers to inflammation of the mucous membranes

(13.3\%), with response durations of 20.0 months and 20.3 months, respectively. No patient in cohort 2 had microsatellite instability-high tumors.

Reductions in target lesion size from baseline were observed in all 24 patients (100\%) who had measurable disease at baseline and $\geq 1$ evaluable postbaseline assessment (Fig. 1a). Responders generally experienced reduction in 
Table 3 Treatment-related AE of any grade occurring in $\geq 10 \%$ of patients and grade 3 treatment-related AEs occurring in $\geq 1$ patient in cohort 3

\begin{tabular}{lll}
\hline Treatment-related AEs ${ }^{\mathrm{a}} \boldsymbol{n}(\boldsymbol{\%})$ & $\begin{array}{l}\text { Cohort } 3 \\
\text { N=31 }\end{array}$ \\
\cline { 2 - 3 } & Any grade & Grade 3 \\
\hline Any & $24(77.4)$ & $6(19.4)$ \\
Fatigue & $8(25.8)$ & 0 \\
Pruritus & $7(22.6)$ & 0 \\
Pneumonitis & $4(12.9)$ & 0 \\
Bile duct obstruction & $1(3.2)$ & $1(3.2)$ \\
Colitis & $1(3.2)$ & $1(3.2)$ \\
Dehydration & $1(3.2)$ & $1(3.2)$ \\
Diffuse uveal melanocytic proliferation & $1(3.2)$ & $1(3.2)$ \\
Hyponatremia & $1(3.2)$ & $1(3.2)$ \\
Neutropenia & $1(3.2)$ & $1(3.2)$ \\
Rash & $1(3.2)$ & $1(3.2)$ \\
\hline
\end{tabular}

$A E$ adverse event

${ }^{a}$ Attribution of AEs to study treatment was determined by the investigator

tumor burden within a few months (Fig. 1b; Online Resource Figure S2).

In cohort 2, 16 patients had PD-L1 CPS $\geq 1$ and eight had PD-L1 CPS $<1$. Among the 16 patients with PD-L1 CPS $\geq 1$, ORR was $68.8 \%$ (95\% CI 41.3-89.0\%; Online Resource Table S10), median time to response was 2.1 months (range 1.9-3.5), and median duration of response was 4.6 months (range 3.2-18.1+). Among the eight patients with PD-L1 CPS $<1$, ORR was $37.5 \%(95 \%$ CI $8.5-75.5 \%$ ), median time to response was 3.6 months (range 2.1-3.7), and median duration of response was 5.4 months (range 2.8-10.1+).

Among 19 patients receiving pembrolizumab, cisplatin, and 5-fluorouracil, ORR was 57.9\% (96\% CI 33.5-79.7\%). Among six Japanese patients receiving pembrolizumab, cisplatin, and capecitabine, ORR was $66.7 \%(95 \% \mathrm{CI}$ 22.3-95.7\%) (Online Resource Table S11).

\section{Cohort 3}

Confirmed ORR was $25.8 \%$ (95\% CI 11.9-44.6\%), with complete response in two patients (6.5\%) (Table 3$)$. Median time to response was 2.1 months (range 1.5-6.2) and median response duration was 9.6 months (range 2.1-17.8+) (Table 3). Response was ongoing at data cutoff in three of eight responders $(37.5 \%)$, with response durations of $17.4,13.7$, and 17.8 months. One patient in cohort 3 had microsatellite instability-high tumors and experienced a partial response.

Of 28 patients with measurable disease at baseline who had $\geq 1$ evaluable postbaseline assessment, $24(85.7 \%)$ experienced reduction in target lesion size from baseline (Fig. 1c). Decrease in tumor burden was generally maintained over several assessments (Fig. 1d; Online Resource Figure S2).

Table 4 Antitumor activity ${ }^{\mathrm{a}}$ assessed by central review per RECIST v1.1 and duration of response

\begin{tabular}{|c|c|c|c|c|}
\hline \multirow[t]{2}{*}{ Category } & \multicolumn{2}{|l|}{$\begin{array}{l}\text { Cohort } 2 \\
N=25\end{array}$} & \multicolumn{2}{|l|}{$\begin{array}{l}\text { Cohort } 3 \\
N=31\end{array}$} \\
\hline & $n$ & $\%\left(95 \% \mathrm{CI}^{\mathrm{b}}\right)$ & $n$ & $\%\left(95 \% \mathrm{CI}^{\mathrm{b}}\right)$ \\
\hline Objective response rate ${ }^{c}$ & 15 & $60.0(38.7-78.9)$ & 8 & $25.8(11.9-44.6)$ \\
\hline Disease control rate $^{\mathrm{d}}$ & 20 & $80.0(59.3-93.2)$ & 11 & $35.5(19.2-54.6)$ \\
\hline \multicolumn{5}{|l|}{ Best overall response } \\
\hline Complete response & 1 & $4.0(0.1-20.4)$ & 2 & $6.5(0.8-21.4)$ \\
\hline Partial response & 14 & $56.0(34.9-75.6)$ & 6 & $19.4(7.5-37.5)$ \\
\hline Stable disease & 8 & $32.0(14.9-53.5)$ & 9 & $29.0(14.2-48.0)$ \\
\hline Progressive disease & 1 & $4.0(0.1-20.4)$ & 12 & $38.7(21.8-57.8)$ \\
\hline Nonevaluable/no assessment & 1 & $4.0(0.1-20.4)$ & 2 & $6.5(0.8-21.4)$ \\
\hline Median (range) time to response (months) & $2.1(1.9-3.7)$ & & $2.1(1.5-6.2)$ & \\
\hline Median (range) duration of response (months) & $4.6(2.6-20.3+)$ & & $9.6(2.1-17.8+)$ & \\
\hline
\end{tabular}

The + indicates that there was no progressive disease at last disease assessment

CI confidence interval, RECIST Response Evaluation Criteria in Solid Tumors

${ }^{a}$ Confirmed by repeat radiographic assessment $\geq 4$ weeks after first documentation of response

${ }^{\mathrm{b}}$ Based on binomial exact CI method

${ }^{\mathrm{c}}$ Complete response + partial response

${ }^{\mathrm{d}}$ Complete response + partial response + stable disease maintained for $\geq 6$ months 

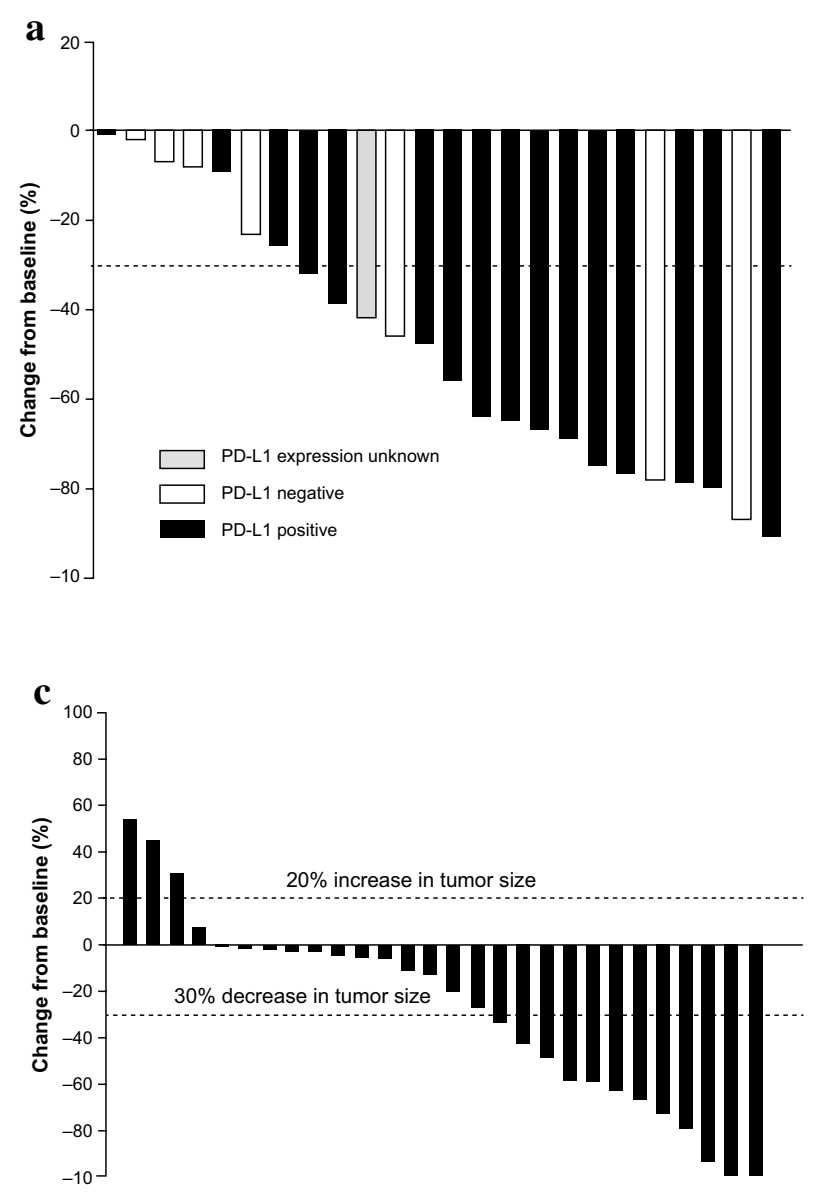

Fig. 1 Antitumor activity. a Best change from baseline in the sum of longest target lesion diameters per patient by PD-L1 expression in cohort $2(n=24)^{\mathrm{a}}$. b Duration of exposure and best response in confirmed responders in cohort $2(n=15)^{\mathrm{b}}$. c Maximum percentage change from baseline in the sum of longest diameter of target lesions per patient $(n=28)^{\mathrm{a}}$. d Duration of exposure and best response in confirmed responders $(n=8)^{\mathrm{b}}$. ${ }^{\mathrm{a}}$ Patients with measurable disease

\section{Survival outcomes}

\section{Cohort 2}

Median PFS was 6.6 months (95\% CI 5.9-10.6), and estimated PFS rate at 6 months was $68.0 \%$ (Fig. 2a). Median OS was 13.8 months (95\% CI 8.6-not estimable), and estimated OS rates were $52 \%$ and $48 \%$ at 12 and 18 months, respectively (Fig. 2b). In patients with PD-L1 CPS $\geq 1$ and $<1$, median OS was 11.1 months (95\% CI 5.4-22.3) and 19.8 months (95\% CI 1.8-not estimable), respectively.
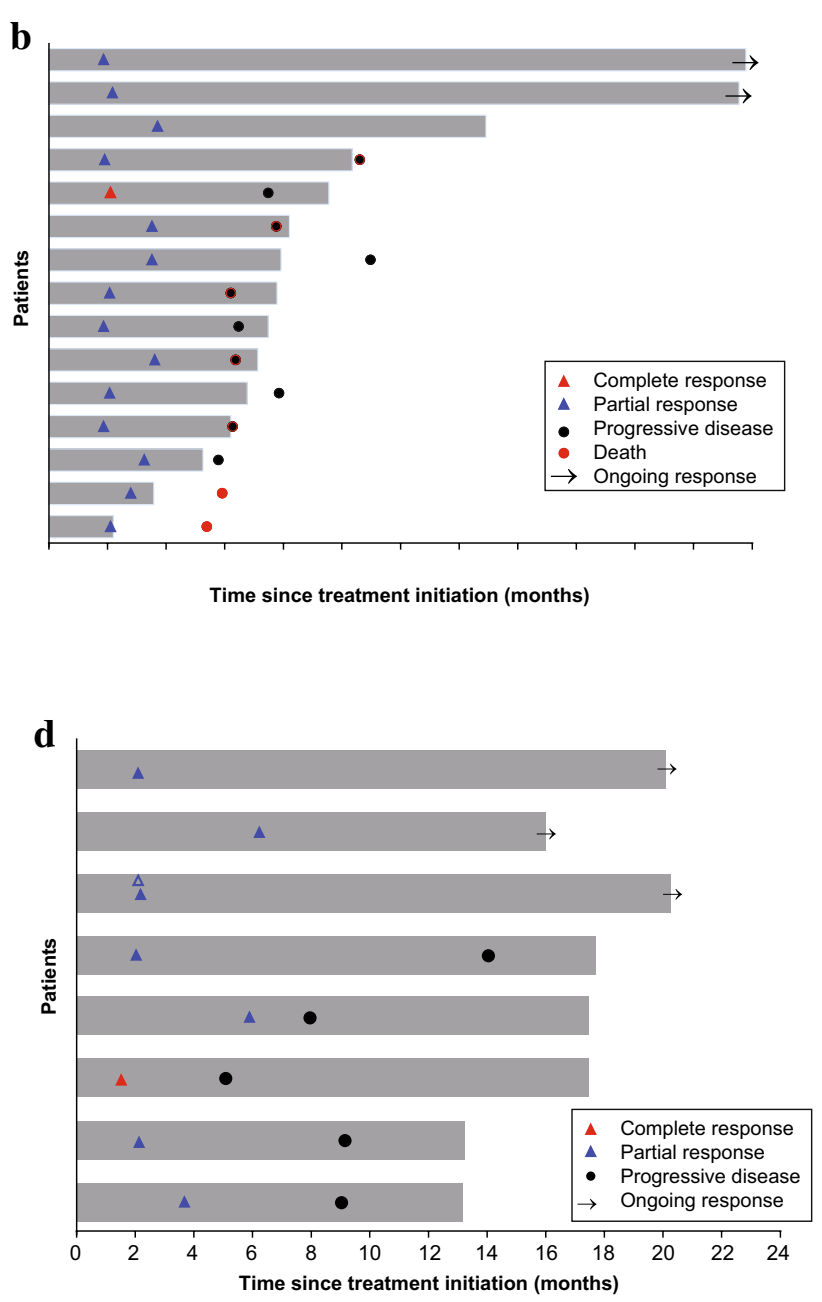

per RECIST v1.1 by central review at baseline who had $\geq 1$ evaluable postbaseline assessment. ${ }^{\mathrm{b}}$ Patients with measurable disease per RECIST $v 1.1$ by central review at baseline who had $\geq 1$ postbaseline assessment and had confirmed response. Bar length indicates time to last dose of study drug. Time to first confirmed response is shown. PD-L1, programmed death ligand 1; RECIST v1.1, Response Evaluation Criteria in Solid Tumors version 1.1

\section{Cohort 3}

Median PFS was 3.3 months (95\% CI 2.0-6.0 months), and estimated PFS rate at 6 months was 34.9\% (Fig. 2c). Median OS was 20.7 months (95\% CI 9.2-20.7), and estimated OS rates were $63 \%$ and $55 \%$ at 12 and 18 months, respectively (Fig. 2d). 

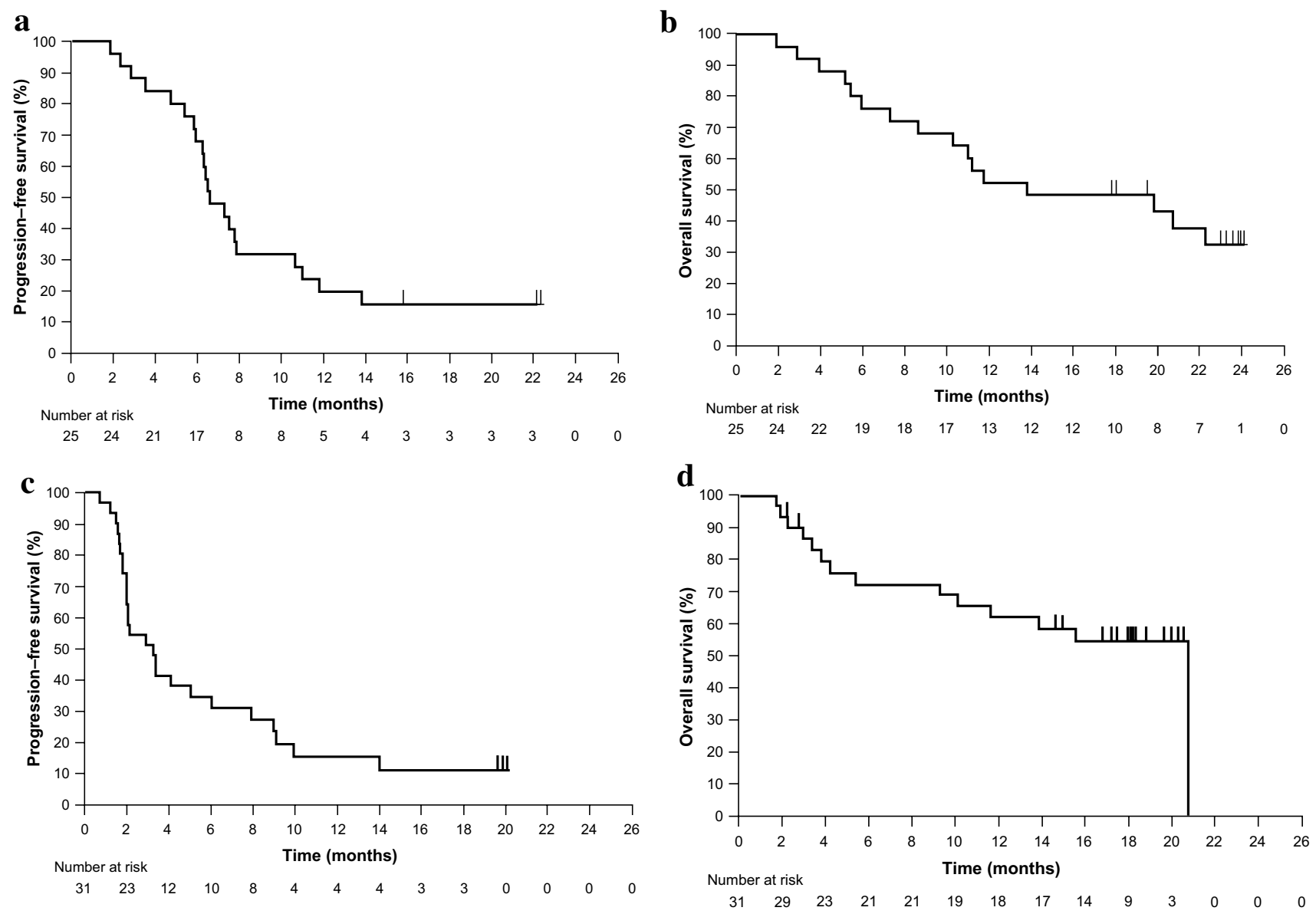

Fig. 2 Kaplan-Meier estimates of a progression-free survival and $\mathbf{b}$ overall survival in cohort 2, and $\mathbf{c}$ progression-free survival and $\mathbf{d}$ overall survival in cohort 3

\section{Discussion}

Cohort 1 of the KEYNOTE-059 study confirmed that pembrolizumab monotherapy provided durable responses with manageable safety in patients with advanced G/GEJ adenocarcinoma that had progressed following second-line treatment [21]. Although patient numbers in the current analyses were small, results from cohorts 2 and 3 of the KEYNOTE-059 study demonstrated manageable safety and antitumor activity for pembrolizumab as monotherapy and as combination therapy with cisplatin plus 5-fluorouracil or capecitabine in previously untreated advanced G/GEJ adenocarcinoma. Although it is difficult to draw definitive conclusions regarding the clinical impact of pembrolizumab based solely on these data, the results, along with those from cohort 1 of the study [21], support the growing body of evidence for the potential use of pembrolizumab in patients with G/GEJ adenocarcinoma. Additional large-scale clinical trials are needed to confirm efficacy and safety in these patients.
In cohort 2, the incidence and severity of TRAEs with pembrolizumab plus cisplatin plus 5-fluorouracil or capecitabine were generally consistent with those of known toxic effects of cisplatin plus fluoropyrimidine [7, $9,10,12]$. The incidence of grades 3 and 4 chemotherapyrelated neutropenia (48\% and $16 \%$, respectively) in this cohort was somewhat higher than expected; previous studies of first-line chemotherapy in patients with advanced gastric cancer have reported grade $\geq 3$ neutropenia occurring at rates of $20-44 \%$ [29-31], although these data were collected from larger study populations that may not be directly comparable. It is notable that neutropenia levels were not elevated in either cohort 3 or cohort 1 [21], but, with a population of just 25 patients, it is difficult to draw definitive conclusions as to the cause of the slightly greater incidence in cohort 2 . The incidence of pembrolizumabrelated stomatitis ( $12 \%$ overall; $8 \%$ grade 3 ) also initially appeared to be higher than expected, but this was likely an artifact given the small number of patients involved (3 of 25). Although a similar incidence of pembrolizumabrelated stomatitis ( 2 of $23 ; 9 \%$ ) was reported in a recently 
published study of patients with advanced colorectal carcinoma [32], the risk associated with pembrolizumab appears to be small compared with the number of patients in whom chemotherapy-related stomatitis develops (52\% in the current study; $16 \%$ grade $\geq 3$ ). Immune-mediated AEs were similar to those reported with pembrolizumab plus platinum-based doublets in non-small cell lung cancer [26], suggesting a degree of constancy across nonhematologic malignancies.

In cohort 3 , the safety profile of pembrolizumab monotherapy was consistent with that previously reported [21, 33]. No new safety signals were identified. Relative to standard chemotherapy, pembrolizumab had lower rates of grade 3/4 AEs and discontinuation because of AEs, suggesting potential for a greater therapeutic index with pembrolizumab than with available therapies [7-12] and the possibility of combining with other therapies.

Although study designs and eligibility criteria differ across trials, the ORR of $60.0 \%$ (95\% CI $39.0-79.0 \%$ ) with pembrolizumab plus cisplatin and 5-fluorouracil or capecitabine in cohort 2 is encouraging relative to response rates observed with cisplatin and fluoropyrimidine doublets (25-48\%) [7-12]. The ORR of $25.8 \%$ (95\% CI $11.9-44.6 \%$ ) with pembrolizumab monotherapy in patients with PD-L1 CPS $\geq 1$ was also within the range expected with chemotherapy (25-48\%) [7-12]. Additionally, median OS in both cohorts compares favorably with the OS with chemotherapy (8-11.2 months), as does 1-year OS [7-12].

Sixty-four percent of patients in cohort 2 were PD-L1 positive (CPS $\geq 1$ ), compared with an overall prevalence of PD-L1 positivity in advanced gastric cancer of 30-65\%, although different methods, antibodies, and cutoff values were used across studies [15-19]. Analysis of patients with PD-L1 CPS $\geq 1$ in cohort 2 suggests that PD-L1 expression enhances response to pembrolizumab plus chemotherapy; however, larger prospective clinical trials are necessary to substantiate these results.

One patient in cohort 3 who had microsatellite instabilityhigh tumors experienced a partial response. Although this partial response contributed to the ORR in cohort 3, it does not account for the ORR in its entirety. This finding is consistent with that reported for pembrolizumab monotherapy in the cohort of patients who have received $\geq 2$ prior therapies for G/GEJ cancer in this KEYNOTE-059 study [21].

Overall, pembrolizumab plus cisplatin and 5-fluorouracil or capecitabine demonstrated manageable safety and promising antitumor activity as first-line therapy in advanced G/GEJ adenocarcinoma, regardless of PD-L1 expression. Pembrolizumab monotherapy also demonstrated encouraging antitumor activity and acceptable safety in patients with PD-L1 CPS $\geq 1$. Results support continued evaluation of pembrolizumab in clinical trials.
Acknowledgements The authors thank the patients and their families and caregivers for participating in the study. Medical writing and/or editorial assistance was provided by Melanie Sweetlove, MSc, and Sarita S. Shaevitz, PhD, of the ApotheCom pembrolizumab team (Yardley, PA, USA). This assistance was funded by Merck Sharp \& Dohme Corp., a subsidiary of Merck \& Co., Inc., Kenilworth, NJ, USA. Funding for this research was provided by Merck Sharp \& Dohme Corp., a subsidiary of Merck \& Co., Inc., Kenilworth, NJ, USA. Merck Sharp \& Dohme Corp., a subsidiary of Merck \& Co., Inc., Kenilworth, NJ, USA, provided pembrolizumab for the study.

Funding Funding for this research was provided by Merck Sharp \& Dohme Corp., a subsidiary of Merck \& Co., Inc., Kenilworth, NJ, USA.

\section{Compliance with ethical standards}

Ethics standards All procedures followed were in accordance with the ethical standards of the responsible committee on human experimentation (institutional and national) and with the Helsinki Declaration of 1964 and later versions. Informed consent to be included in the study, or the equivalent, was obtained from all patients.

Conflict of interest Y-JB: consultant or advisory role: AstraZeneca, Novartis, Roche, Genentech, Merck Sharp \& Dohme, Pfizer, Bayer, Bristol-Myers Squibb, Eli Lilly, Merck Serono, Five Prime, Merrimack, Taiho, Ono, ADC Therapeutics, Green Cross, Samyang Biopharma; research funding (institution): AstraZeneca, Novartis, Roche/ Genentech, Merck Sharp \& Dohme, Merck Serono, Pfizer, Bayer, Bristol-Myers Squibb, GlaxoSmithKline, Eli Lilly, Boehringer Ingelheim, MacroGenics, Boston Biomedical, Five Prime, Chong Kun Dang Bio, Ono pharmaceutical, Otsuka, Taiho, Takeda, BeiGene, Hanmi, Green Cross, Curis. Y-KK: consultant or advisory role: Bristol-Myers Squibb, Ono Pharmaceutical, Daehwa, Novartis; research funding: LSK Biopharma. DVC: consultant or advisory role: Merck, BristolMyers Squibb, Eli Lilly, Genentech/Roche, Amgen, Taiho, Five Prime. KM: honoraria: Chugai Pharmaceutical, Takeda Pharmaceutical, Eli Lilly Japan K.K., Merck Serono, Taiho, Yakult Honsha; research funding to institution: Shionogi \& Co., Ltd., Merck Sharp \& Dohme, K.K., Daiichi Sankyo, Kyowa Hakko Kirin Co. Ltd., Gilead Sciences. CSF: consultant or advisory role: Agios, Bain Capital, Bayer, Celgene, Dicerna, Five Prime Therapeutics, Gilead Sciences, Eli Lilly, Entrinsic Health, Genentech, KEW, Merck, Merrimack Pharmaceuticals, Pfizer, Sanofi, Taiho, and Unum Therapeutics. He also serves as a Director for CytomX Therapeutics and owns unexercised stock options for CytomX and Entrinsic Health. RG: honoraria: Bristol-Myers Squibb, Eli Lilly, Medison, Roche, Novartis, Janssen; consulting or advisory role: Bayer, Merck Sharp \& Dohme, Novartis; travel, accommodation, expenses: Roche, Bristol-Myers Squibb. HH: consultant or advisory role: Ono Pharmaceutical, Chugai Pharmaceutical; research funding (institution): AstraZeneca, Chugai Pharmaceutical, Merck Serono, Merck Sharp \& Dohme, Ono Pharmaceutical, Taiho Pharmaceutical, Takeda, Boehringer Ingelheim, Sumitomo Dainippon Pharma, Daiichi Sankyo, Eli Lilly; TG: research funding (institution): AstraZeneca. Merck Sharp \& Dohme consultant or advisory role: AstraZeneca and AbbVie. SIJ: research funding (institution): AstraZeneca. MG: consultant or advisory role: Merck Sharp \& Dohme, Eli Lilly. CB: honoraria: Bristol-Myers Squibb, Eli Lilly, Amgen, Merck Sharp \& Dohme; consultant or advisory role: Roche, Servier; research funding (institution): Roche. TD: consultant or advisory role: Eli Lilly, Chugai Pharmaceutical, Kyowa Hakko Kirin Co., Novartis, Merck Sharp \& Dohme, Daiichi Sankyo, Amgen; research funding (institution): Taiho, Novartis, Merck Serono, Astellas, Merck Sharp \& Dohme, Janssen, Boehringer Ingelheim, Takeda, Pfizer, Eli Lilly, Sumitomo Group, Chugai Pharmaceutical, Bayer, Kyowa Hakko Kirin Co., Daiichi Sankyo, Celgene. 
HY: research funding (institution): Eli Lilly, Genentech; honoraria (institution): Eli Lilly, Genentech, Astellas, Five Prime Therapeutics, LSK Biopharma. MJS, SS, and JW: employee: Merck Sharp \& Dohme Corp., a subsidiary of Merck \& Co., Inc., Kenilworth, NJ, USA. RPD: former employee: Merck Sharp \& Dohme Corp., a subsidiary of Merck \& Co., Inc., Kenilworth, NJ, USA. ZAW: consultant or advisory role: Genentech, Array Biopharma, Sirtex, Novartis, Five Prime Therapeutics. HCC: consultant or advisory role: Taiho, Celltrion, Merck Sharp \& Dohme, Eli Lilly, Quintiles, Bristol-Myers Squibb; speaker's bureau: Merck Serono, Eli Lilly, Foundation Medicine; research funding (institution): Eli Lilly, GlaxoSmithKline, Merck Sharp \& Dohme, Merck Serono, Bristol-Myers Squibb/Ono Pharmaceutical, Taiho.

OpenAccess This article is distributed under the terms of the Creative Commons Attribution 4.0 International License (http://creativeco mmons.org/licenses/by/4.0/), which permits unrestricted use, distribution, and reproduction in any medium, provided you give appropriate credit to the original author(s) and the source, provide a link to the Creative Commons license, and indicate if changes were made.

\section{References}

1. Ferlay J, Soerjomataram I, Dikshit R, Eser S, Mathers C, Rebelo $\mathrm{M}$, et al. Cancer incidence and mortality worldwide: sources, methods and major patterns in GLOBOCAN 2012. Int J Cancer. 2015;136:E359-86.

2. Shah MA. Update on metastatic gastric and esophageal cancers. J Clin Oncol. 2015;33:1760-9.

3. Goode EF, Smyth EC. Immunotherapy for gastroesophageal cancer. J Clin Med. 2016;5:84

4. National Comprehensive Cancer Network. NCCN clinical practice guidelines in oncology (NCCN guidelines): gastric cancer. (Version 2. 2018). https://www.nccn.org/professionals/physician_gls/ pdf/gastric.pdf. 2018. Accessed 31 Oct 2018.

5. Smyth EC, Verheij M, Allum W, Cunningham D, Cervantes A, Arnold D, ESMO Guidelines Committee. Gastric cancer: ESMO Clinical Practice Guidelines for diagnosis, treatment and followup. Ann Oncol. 2016;27(suppl 5):v38-49.

6. National Comprehensive Cancer Network. NCCN clinical practice guidelines in oncology (NCCN guidelines): esophageal and esophagogastric junction cancers. (Version 2. 2018). https://www. nccn.org/professionals/physician_gls/pdf/esophageal.pdf. 2018. Accessed 31 Oct 2018.

7. Dank M, Zaluski J, Barone C, Valvere V, Yalcin S, Peschel C, et al. Randomized phase III study comparing irinotecan combined with 5-fluorouracil and folinic acid to cisplatin combined with 5-fluorouracil in chemotherapy naive patients with advanced adenocarcinoma of the stomach or esophagogastric junction. Ann Oncol. 2008;19:1450-7.

8. Al-Batran SE, Hartmann JT, Probst S, Schmalenberg H, Hollerbach S, Hofheinz R, et al. Phase III trial in metastatic gastroesophageal adenocarcinoma with fluorouracil, leucovorin plus either oxaliplatin or cisplatin: a study of the Arbeitsgemeinschaft Internistische Onkologie. J Clin Oncol. 2008;26:1435-42.

9. Ajani JA, Rodriguez W, Bodoky G, Moiseyenko V, Lichinitser $\mathrm{M}$, Gorbunova V, et al. Multicenter phase III comparison of cisplatin/S-1 with cisplatin/infusional fluorouracil in advanced gastric or gastroesophageal adenocarcinoma study: the FLAGS trial. J Clin Oncol. 2010;28:1547-53.

10. Van Cutsem E, Moiseyenko VM, Tjulandin S, Majlis A, Constenla $\mathrm{M}$, Boni C, et al. Phase III study of docetaxel and cisplatin plus fluorouracil compared with cisplatin and fluorouracil as first-line therapy for advanced gastric cancer: a report of the V325 Study Group. J Clin Oncol. 2006;24:4991-7.

11. Cunningham D, Starling N, Rao S, Iveson T, Nicolson M, Coxon F, et al. Capecitabine and oxaliplatin for advanced esophagogastric cancer. N Engl J Med. 2008;358:36-46.

12. Kang YK, Kang WK, Shin DB, Chen J, Xiong J, Wang J, et al. Capecitabine/cisplatin versus 5-fluorouracil/cisplatin as first-line therapy in patients with advanced gastric cancer: a randomised phase III noninferiority trial. Ann Oncol. 2009;20:666-73.

13. Francisco LM, Sage PT, Sharpe AH. The PD-1 pathway in tolerance and autoimmunity. Immunol Rev. 2010;236:219-42.

14. Pardoll DM. Immunology beats cancer: a blueprint for successful translation. Nat Immunol. 2012;13:1129-32.

15. Geng Y, Wang H, Lu C, Li Q, Xu B, Jiang J, et al. Expression of costimulatory molecules B7-H1, B7-H4 and Foxp3+ Tregs in gastric cancer and its clinical significance. Int J Clin Oncol. 2015;20:273-81.

16. Hou J, Yu Z, Xiang R, Li C, Wang L, Chen S, et al. Correlation between infiltration of FOXP3+ regulatory T cells and expression of B7-H1 in the tumor tissues of gastric cancer. Exp Mol Pathol. 2014;96:284-91.

17. Boger C, Behrens HM, Mathiak M, Krüger S, Kalthoff H, Röcken $\mathrm{C}$, et al. PD-L1 is an independent prognostic predictor in gastric cancer of Western patients. Oncotarget. 2016;7:24269-83.

18. Qing Y, Li Q, Ren T, Xia W, Peng Y, Liu GL, et al. Upregulation of PD-L1 and APE1 is associated with tumorigenesis and poor prognosis of gastric cancer. Drug Des Devel Ther. 2015;9:901-9.

19. Wu C, Zhu Y, Jiang J, Zhao J, Zhang XG, Xu N. Immunohistochemical localization of programmed death-1 ligand-1 (PD-L1) in gastric carcinoma and its clinical significance. Acta Histochem. 2006;108:19-24.

20. Merck Sharp \& Dohme Corp. KETRUDA ${ }^{\circledR}$ (pembrolizumab), for injection, for intravenous use. Whitehouse Station; November 2018. https://www.merck.com/product/usa/pi_circulars/k/keytr uda/keytruda_pi.pdf. Accessed 12 Feb 2019

21. Fuchs CS, Doi T, Jang RW, Muro K, Satoh T, Machado M, et al. Safety and efficacy of pembrolizumab monotherapy in patients with previously treated advanced gastric and gastroesophageal junction cancer: phase 2 clinical KEYNOTE-059 Trial. JAMA Oncol. 2018;4:e180013.

22. Shitara K, Özgüroğlu M, Bang Y-J, Di Bartolomeo M, Mandalà M, Ryu MH, et al. Pembrolizumab versus paclitaxel for previously treated, advanced gastric or gastro-oesophageal junction cancer (KEYNOTE-061): a randomised, open-label, controlled, phase 3 trial. Lancet. 2018;392:123-33.

23. Zitvogel L, Galluzzi L, Smyth MJ, Kroemer G. Mechanism of action of conventional and targeted anticancer therapies: reinstating immunosurveillance. Immunity. 2013;39:74-88.

24. Wan S, Pestka S, Jubin RG, Lyu YL, Tsai YC, Liu LF. Chemotherapeutics and radiation stimulate MHC class I expression through elevated interferon-beta signaling in breast cancer cells. PLoS One. 2012;7:e32542.

25. Ramakrishnan R, Assudani D, Nagaraj S, Hunter T, Cho HI, Antonia $S$, et al. Chemotherapy enhances tumor cell susceptibility to CTL-mediated killing during cancer immunotherapy in mice. J Clin Invest. 2010;120:1111-24.

26. Langer CJ, Gadgeel SM, Borghaei H, Borghaei H, Papadimitrakopoulou VA, Patnaik A, et al. Carboplatin and pemetrexed with or without pembrolizumab for advanced, non-squamous non-smallcell lung cancer: a randomised, phase 2 cohort of the open-label KEYNOTE-021 study. Lancet Oncol. 2016;17:1497-508.

27. Lara P, Beckett L, Li Y, Parikh M, Robles D, Aujla P, et al. Combination checkpoint immunotherapy and cytotoxic chemotherapy: pembrolizumab (Pembro) plus either docetaxel or gemcitabine 
in patients with advanced or metastatic urothelial cancer. J Clin Oncol. 2017. https://doi.org/10.1200/JCO.2017.35.6_suppl.398

28. Clipper CJ, Pearson ES. The use of confidence or fiducial limits illustrated in the case of binomial. Biometrika. 1934;26:404-13.

29. Pinto C, Di Fabio F, Barone C, Siena S, Falcone A, Cascinu S, et al. Phase II study of cetuximab in combination with cisplatin and docetaxel in patients with untreated advanced gastric or gastro-oesophageal junction adenocarcinoma (DOCETUX study). Br J Cancer. 2009;101:1261-8.

30. Lordick F, Kang YK, Chung HC, Salman P, Oh SC, Bodoky $\mathrm{G}$, et al. Capecitabine and cisplatin with or without cetuximab for patients with previously untreated advanced gastric cancer (EXPAND): a randomised, open-label phase 3 trial. Lancet Oncol. 2013;14:490-9.
31. Yamada Y, Higuchi K, Nishikawa K, Gotoh M, Fuse N, Sugimoto N, et al. Phase III study comparing oxaliplatin plus S-1 with cisplatin plus S-1 in chemotherapy-naive patients with advanced gastric cancer. Ann Oncol. 2015;26:141-8.

32. O'Neil BH, Wallmark JM, Lorente D, Elez E, Raimbourg J, Gomez-Roca C, et al. Safety and antitumor activity of the antiPD-1 antibody pembrolizumab in patients with advanced colorectal carcinoma. PLoS One. 2017;12:e0189848.

33. Muro K, Chung HC, Shankaran V, Geva R, Catenacci D, Gupta S, et al. Pembrolizumab for patients with PD-L1-positive advanced gastric cancer (KEYNOTE-012): a multicentre, open-label, phase 1b trial. Lancet Oncol. 2016;17:717-26.

\section{Affiliations}

\section{Yung-Jue Bang ${ }^{1} \cdot$ Yoon-Koo Kang ${ }^{2} \cdot$ Daniel V. Catenacci $^{3} \cdot$ Kei Muro $^{4} \cdot$ Charles S. Fuchs $^{5} \cdot$ Ravit Geva $^{6} \cdot$ Hiroki Hara $^{7}$. Talia Golan $^{8} \cdot$ Marcelo Garrido $^{9}$. Shadia I. Jalal ${ }^{10}$. Christophe Borg ${ }^{11} \cdot$ Toshihiko Doi $^{12} \cdot$ Harry H. Yoon $^{13}$. Mary J. Savage ${ }^{14}$. Jiangdian Wang ${ }^{14} \cdot$ Rita P. Dalal $^{14} \cdot$ Sukrut Shah $^{14} \cdot$ Zev A. Wainberg ${ }^{15} \cdot$ Hyun Cheol Chung $^{16}$}

1 Department of Internal Medicine, Seoul National University College of Medicine, 101, Daehak-ro, Jongno-gu, Seoul 03080, Republic of Korea

2 Division of Oncology, Department of Internal Medicine, Asan Medical Center, University of Ulsan, Seoul, Republic of Korea

3 Section of Hematology/Oncology, Department of Medicine, University of Chicago Medicine, Chicago, IL, USA

4 Department of Clinical Oncology, Aichi Cancer Center Hospital, Nagoya, Aichi, Japan

5 Department of Medical Oncology, Yale Cancer Center, New Haven, CT, USA

6 Department of Oncology, Tel-Aviv Sourasky Medical Center, Tel Aviv University, Tel Aviv, Israel

7 Department of Gastroenterology, Saitama Cancer Center, Saitama, Japan

8 Department of Oncology, The Oncology Institute at the Chaim Sheba Medical Center, Sackler Faculty of Medicine, Tel Aviv University, Tel Aviv, Israel
9 Hematology and Medical Oncology, Pontificia Universidad Católica de Chile, Santiago, Chile

10 Department of Internal Medicine, Indiana University School of Medicine, Indianapolis, IN, USA

11 Department of Medical Oncology, University Hospital of Besançon, Besancon, France

12 Department of Gastrointestinal Oncology, National Cancer Center Hospital East, Chiba, Kashiwa, Japan

13 Department of Medical Oncology, Mayo Clinic, Rochester, MN, USA

14 Medical Oncology, Merck \& Co., Inc., Kenilworth, NJ, USA

15 Division of Hematology Oncology, David Geffen School of Medicine at UCLA, Los Angeles, CA, USA

16 Division of Medical Oncology, Yonsei Cancer Center, Yonsei University College of Medicine, Seoul, Republic of Korea 